\title{
ENERGY AND STRUCTURE OF [100] ALUMINIUM GRAIN BOUNDARY
}

\author{
A. OTSUKI, H. ISONO(1) and M. MIZUNO \\ Department of Metal Science and Technology, Faculty of Engineering. \\ Kyoto University, Kyoto 606, Japan
}

\begin{abstract}
The energy and structure of the [100] twist and tilt grain boundaries in aluminium have been studied by fracture stress and dihedral angle measurements. The relative boundary energy was estimated as a function of misorientation. The fracture stress and the dihedral angle show a discontinuous change at a temperature, suggesting a drastic change in the grain boundary energy at this temperature. This transition temperature was measured as a function of misorientation.
\end{abstract}

\section{Introduction}

The grain boundary in a crystal has long been discussed both theoretically and experimentally. Recently, computer simulations of the grain boundary structure have been made by many authors using a pairweis interaction model. Among them, the structure unit model seems to give a better understanding of the grain boundary structure $[1,2]$. The structure unit model is supported by the observation of strain contrast images produced by a periodic structure of the grain boundary core region $[4,5]$.

According to the structure unit model[1,2], a high $\Sigma$ boundary consists of two fundamental structure units forming low $\Sigma$ boundaries, where the symbol $\Sigma$ shows the reciprocal density of the coincidence sites. If a high $\sum$ boundary has a periodic ordered structure with two kinds of fundamental structure units, this boundary is called the "delimiting" boundary, which divides the misorientation or inclination into several ranges. If the delimiting boundary is composed of the sequence of one fundamental structure unit, this boundary is called the "favored" boundary. An Intervening boundary with a misorientation between those of two delimiting boundaries is composed of distorted units of delimiting boundaries. Since the distorted unit can be regarded as grain boundary dislocations, which originate in the primary lattice and the displacement shift complete (DSC-) lattice in delimiting boundaries. Since the intervening boundary with grain boundary dislocations has a higher boundary energy than that of the delimiting boundary, an energy cusp is expected at a delimiting boundary in the energy vs. misorientation curve.

(1)Present address: Sharp Corp., Rekimoto-cho, Tenri, 632, Japan. 
The energy cusp is expected to give important information on the delimiting boundary[3]. By applying the structure unit model to the [100] tilt boundary of $\mathrm{Cu}$, the grain boundary structure has been well described by the five structure units which classify the grain boundary into three regions depending upon the misorientation $[6,7]$. No energy cusp in the [100] $\mathrm{Cu}$ or $\mathrm{Al}$ tilt boundary has been observed even in the vicinity of very short period low $\Sigma(e . g, \Sigma 5)$ boundaries [8,9] and delimiting boundaries are not also determined from the energy dependence on misorientation.

On the other hand, the energy cusps in the [100] twist boundary of aluminium are clearly observed at $\Sigma 5$ and $\Sigma 13$ misorientations.

Furthermore, the grain rotation experiment showed some indications of other energy cusps[10], while a computer calculation indicates a smaller energy of $\Sigma 25$ coincidence boundary than that of $\Sigma 5$ or $\Sigma 13$ misorientations[11]. It is, therfore, desired to have more detailed experiments of the delimiting boundaries on the [100] twist boundary.

Since both of delimiting and favored boundaries may show energy cusps, an energy cusp does not always indicate the favored boundary. It should be examined whether the intervening boundaries at both sides of a delimiting boundary misorientation have different structure unit components each other. Fortunately, the grain boundary energy in lead has been found to change discontinuously at a specific temperature, suggesting some change of the grain boundary structure[12]. In a previous paper[8] we have reported that this transition temperature depends on the misorientation and it changes discontinuosly at $\Sigma 3$ and ¿11 misorientations on the [110] tilt boundary. Since the examination on the transition temperature of the boundary energy can be compared the structure of delimiting boundary with that of intervening boundaries, a delimiting boundary will be classified whether a favored boundary or not.

In this work, the relative boundary energies of the Al [100] tilt and twist grain boundaries were estimated from the dihedral angle at $240^{\circ} \mathrm{C}(\mathrm{T} / \mathrm{Tm}=0.55$, Tm is the bulk melting temperature expressed in the absolute temperature), while the transition temperature was measured as functions of misorientation and impurity level.

\section{Experimental Methods}

Aluminium [100] bicrystals with desired orientations were made by a Bridgman method using two seed crystals with the growth rate of 15 $\mathrm{mm} / \mathrm{hr}$. The deviation from the ideal relative orientation between two single crystals was within two degrees. Alumininum mother metals with the impurity levels of 3 and $9 \mathrm{ppm}$ were used. The dihedral angle was evaluated from the angle in the grain boundary groove at the Al (s)/Sn$20 \mathrm{zn}(1)$ interface by an optical microscope after vacuum annealing for 6 hrs to 4 days between $210^{\circ}-350^{\circ} \mathrm{C}$.

A specimen with a gauge length of $2.4 \mathrm{~mm}$ and a cross section of $2 \times 2 \mathrm{~mm}^{2}$ for tensile tests was cut from the bicrystal in such a way that its grain boundary plane is normal to the stress axis. First, the specimen was annealed for $1 \mathrm{~min}$ to 3 days at each testing temperature between $210^{\circ}-500^{\circ} \mathrm{C}$ in air. After the gauge portion of the specimen was wet with a Sn-20mass\% $\mathrm{n}$ melt, the tensile test was carried out within $1 \mathrm{~min}$ under an initial strain rate of $0.07 \mathrm{~s}^{-1}$.

\section{Results and Discussion}

3.1 Variation of the grain boundary energy with misorientation

It has been generaliy accepted that the grain boundary energy, $\gamma g b$, is given by the dihedral angle, $\alpha$, as $\gamma_{g b}=2 \gamma_{s} l \cdot \cos (\alpha / 2)$, where $\gamma s$ is the solid liquid interfacial energy. Assuming that $\gamma s 1$ is independent of misorientation, we have the relative $\gamma g b / \gamma s l$ value from observed $\alpha$. The observed dependence of $\alpha$ on the misorientation $\vartheta$ is 
shown in Fig.1, where $\vartheta$ is defined as the angle between $\langle 110\rangle$ directions of two grains. The average energy ratio of the high angle twist boundary to that of the tilt boundary is nearly 0.82 and 1 arger than 0.77 for $\mathrm{Cu}[9]$. The dotted lines show the Read-Shockley relation[13] for small angle boundary regions. The ranges of small angle twist and tilt boundaries are up to roughly $15^{\circ}$ and $20^{\circ}$ and narrower than that of $\mathrm{Cu}[9]$.

The energy of the twist boundary is smaller than that of $\Sigma 1(110)$ small angle tilt boundary and approximately equal to that of $\sum 1^{*}(100)$ small angle tilt boundary. The energy cusps are observed at $\Sigma 61$ a (or $\sum 101\left(11.42^{\circ}\right)$ ) and $\sum 65$ a misorientations with larger $\alpha$ values than dotted line but not at $\Sigma 25 a\left(16.25^{\circ}\right)$ or $\Sigma 41 \mathrm{a}\left(12.68^{\circ}\right)$ or $\Sigma 37 \mathrm{a}\left(18.92^{\circ}\right)$. With Eurther increase in $\vartheta$, the twist boundary shows cusps at $\sum 13 a, \sum 17 a$, $\sum 65 \mathrm{~b}$ and $\Sigma 5$ misorientations and nearly the $\Sigma 29 \mathrm{a}$ misorientation. The wide cusp near $\Sigma 29$ a misorientation may be the cusps composed of $\Sigma 29$ a $\left(43.60^{\circ}\right), \sum 73 \mathrm{c}\left(41.11^{\circ}\right)$ and $\sum 97 \mathrm{C}\left(42.07^{\circ}\right)$ misorientations. The energy cusps in $\sum 5$ and $\Sigma 13$ are broad, probably because high $\sum$ boundaries, such as $\Sigma 109\left(33.40^{\circ}\right)$ or $\sum 185\left(34.21^{\circ}\right)$ exist near the $\Sigma 5$ energy cusps and nearly the $\Sigma 13$ cusp we have $\Sigma 125\left(20.61^{\circ}\right)$ and $\Sigma 149\left(20.02^{\circ}\right)$ boundaries.

The depth of each cusp is roughly constant being independent of $\Sigma$ value or the density of coincidence site in the boundary plane, $\Gamma$, as pointed out by Goodhew[14]. Bristowe and Crocker have shown from calculations of energies of [100] twist boundary of $\mathrm{Cu}$ that the energies of coincidence boundaries decrease in the order of $\Sigma 5, \Sigma 13$ and $\Sigma 25[11]$.

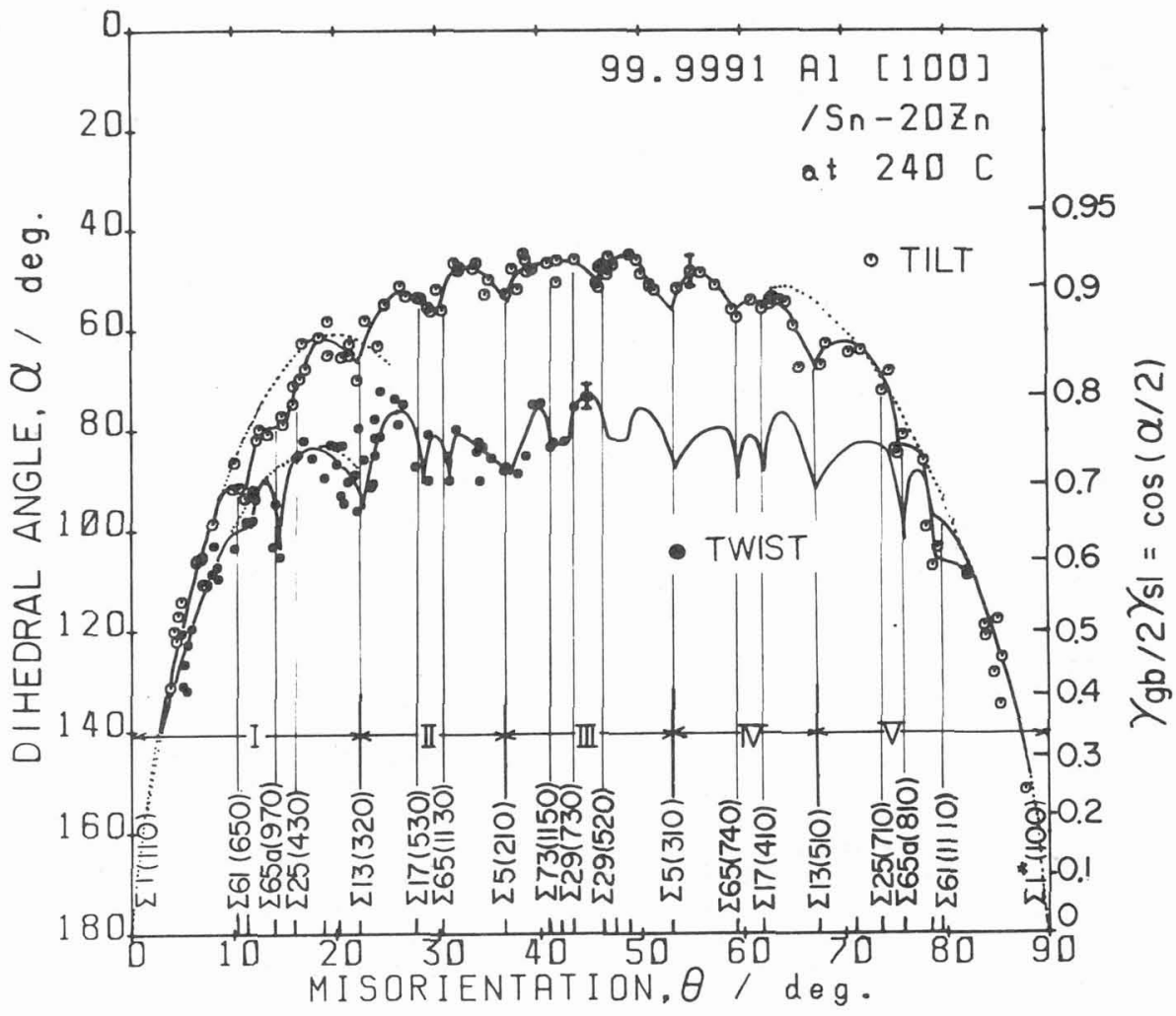

Fig.1 Misorientation dependence of the dihedral angle of the Al [100] twist and tilt boundaries. 
This result is explained by the relaxation of individual atoms inside the larger coincidence site unit cell such as the $\Sigma 13$ in the high $\Sigma$ misorientation. Sutton showed that these relaxed $\Sigma 13$ or $\Sigma 25$ boundaries can be composed of $10 w \Sigma 5$ and $\Sigma 1$ and filler structure units, describing low energy[15]. Hence, cusps of these high $\sum$ boundaries such as $\Sigma 65 a$ or $\sum 65 b$ probably support the structure unit model that high $\Sigma$ twist boundaries are composed of structure units of low $\Sigma$ boundaries such as $\sum 1$ or $\sum 5$.

In the tilt boundary, the energy of the $\Sigma 1^{*}(100)$ small angle boundary is somewhat lower than that of the $\Sigma 1$ (110) small angle boundary. Energy cusps are observed at $\sum 61$ as (or $\sum 101\left(11.42^{\circ}\right.$ ) and $\sum 65 a s$ misorientations of which $\alpha$ values are larger than dotted lines. With further increase in $\vartheta$, the boundary energy increases slightly. In the high angle boundary, energy cusps are clearly observed at $\Sigma 13$ as and $\Sigma 5 \mathrm{~s}$. A cusp is observed at $\Sigma 65 \mathrm{~b}$ misorientation instead of $\sum 17 \mathrm{a}$. The cusps in the tilt boundary are well-defined but shallower than those of the twist boundary. The misorientation dependence of energy is approximately symmetric with respect to $\vartheta=45^{\circ}$.

As observed in the twist boundary, energy cusps in the tilt boundary are also observed in long period high $\sum$ misorientations such as $\Sigma 61 \mathrm{a}, \Sigma 65 \mathrm{a}$ and $\Sigma 65 \mathrm{~b}$. These results support the structure unit model, because the long period high $\sum$ boundary may be composed of short period low $\sum$ boundaries rather than the intrinsic long range structure of high $\Sigma$ boundary. These coincidence boundaries with the cusp may be the delimiting boundary. These boundaries cannot be classified as the "favored" boundary only by observing the cusp. According to the structure unit model[6], we assume that, $\Sigma 1, \Sigma 1 *$ and $\Sigma 5 \mathrm{~s}$ misorientations in the [100] tilt boundary are favored boundary. Besides these boundaries, $\Sigma 13$ as misorientations may be the limits of between the small and the high angle rigeons. This assumption allows us to divide the [100]tilt boundary into 5 regions depending upon the misorientation as indicated by $I, \mathbb{I}, \ldots . V$. in Fig. 1 .

\subsection{Temperature dependence of fracture stress}

We have measured the fracture stress of the sample, because it may give another information about the grain boundary energy. It is well known that the fracture stress increases with decreasing the grain boundary energy[8]. Fig.2 shows the temperature dependence of the fracture stress $\sigma_{F}$ of the $[100]$ tilt boundary for several $\vartheta$. Between $200^{\circ} \mathrm{C}$ and the ductile to brittle transition (DBT) temperature shown by an arrow, the [100] tilt boundary shows the brittle fracture at the grain boundary. Beyond the DBT temperature, the fracture stress is approximately equal to the ultimate tensile stress of the specimen unwet by liquid metal and it decreases with increasing temperature. In the brittle temperature range, the fracture stress abruptly decreases at a temperature, D, and again abruptly increases at $U$ with increasing temperature.

Since the change in the $\gamma \mathrm{sl}$ in the Al/Sn system is continuous between $232^{\circ}$ and $400^{\circ} \mathrm{C}[16]$, the temperature variation of $\mathrm{Ysl}$ in the Al/Sn$20 \mathrm{zn}$ system would also be smooth. The observed temperature variation of the fracture stress, therefore, can be regarded as the grain boundary energy change with temperature. Since $U$ and $D$ are well-defined at roughly the same temperatures for $\theta=14^{\circ}-20^{\circ}$, these boundaries may have the same structure and they change drastically at these transition temperatures, $\mathrm{D}$ and $\mathrm{U}$. The height of steps, on the other hand, decreases with increasing misorientation and for $\vartheta=22^{\circ}$ ( $\left.\sum 13\right)$ the transition temperature becomes unclear. The decrease in the height of steps probably means a decrease of the density of structures attributable to these steps.

We have observed no step when the misorientation increases to $\vartheta=$ $32^{\circ}$. This means that in the region II the grain boundary structure is different from those in the region I (see Fig.1). The boundary with $\vartheta=$ 


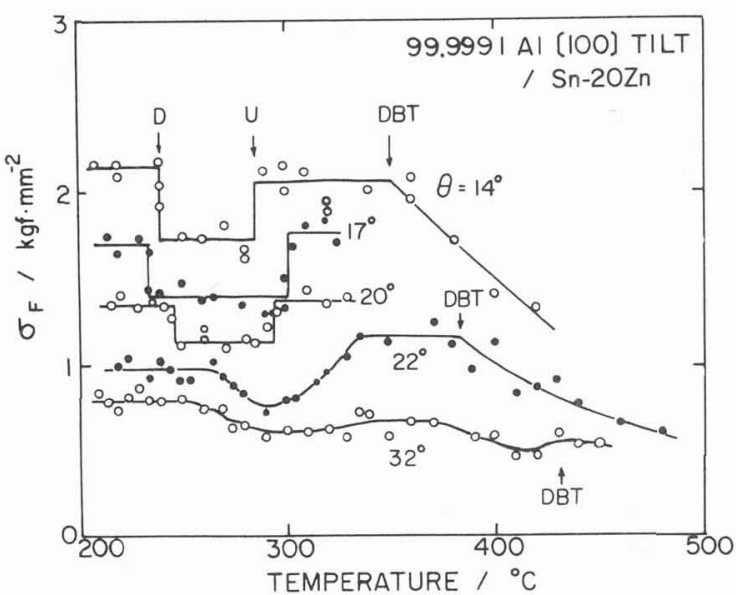

Fig.2 Temperature dependence of the fracture stress of the Al [100] tilt boundary.

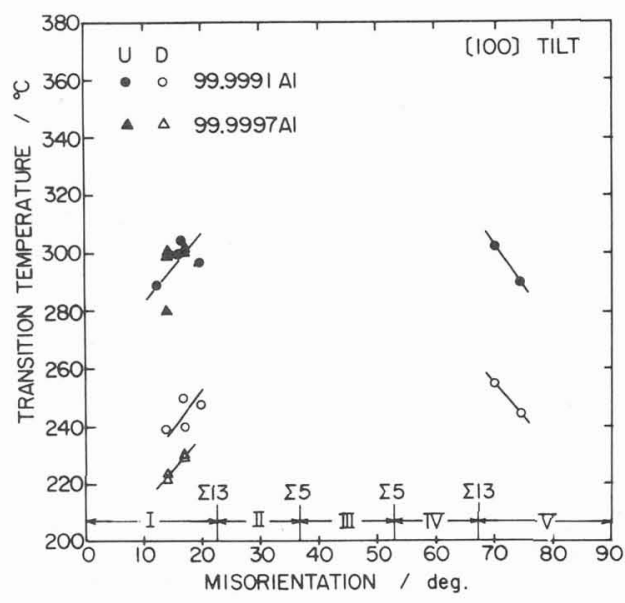

Fig. 3 Misorientation dependence of the transition temperatures, $U$ and $D$ (see text).

$14^{\circ}$ ( $\left.\Sigma 65 a\right)$ is the delimiting boundary because of the energy cusp as shown in Fig.1. As mentioned in the above section the high $\sum$ boundary such as 565 a may be composed of multiple structure units. The structure change of intervening boundary between $\Sigma 65 \mathrm{a}$ and $\Sigma 13 \mathrm{a}$ misorientation may be ascribed to the change of structure or array of structure units in the small angle boundary, because these change is not observed at $8=22^{\circ}$ ( $\Sigma 13 a)$ misorientation. The region of small angle boundary of $\mathrm{Cu}$ exends up to $45^{\circ}[9]$. The electron microscopic observation of high angle $[100]$ tilt boundaries $\left(\vartheta=0^{\circ}\right.$ to $28^{\circ}$ or $38^{\circ}$ ) of $\mathrm{Au}$ showed the strain contrast pattern originated from primary lattice relaxation [4. 5]. On the other hand, since the small angle boundary of Al ranges between $0^{\circ}$ and $20^{\circ}$, the $\sum 13 \mathrm{a}\left(22.62^{\circ}\right)$ and increasing misorientation from $\Sigma 13$ a may not be composed of the $\Sigma 1$ structure unit component.

The temperature dependence of the fracture stress in the region $\nabla$ also shows similar behavior to that in the region $I$ as shown in Fig. 3 . The temperatures $D$ and $U$ in these regions increase with increasing misorientation. The transition temperature $D$ increases with increasing impurity level of aluminium, while the temperature $U$ does not change by the impurity level. In addition to impurity atoms in $\mathrm{Al}$ mother metals, $\mathrm{Sn}$ and $\mathrm{Zn}$ atoms diffused into the grain

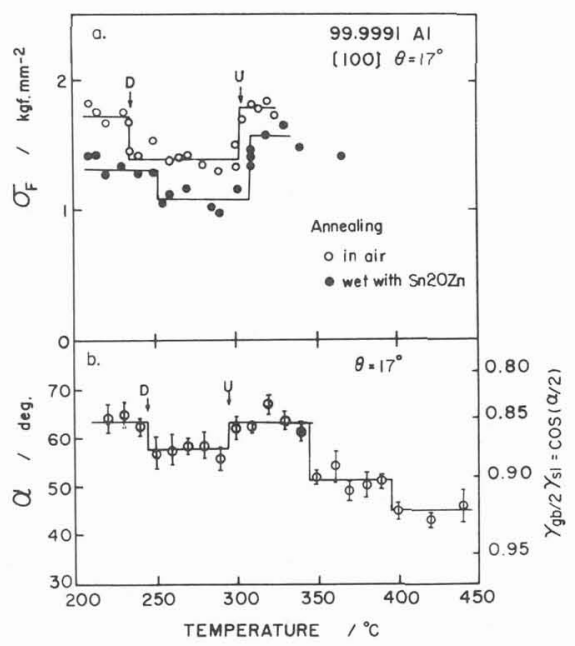

Fig.4 Temperature dependence of $a$ : the fracture stress, $\mathrm{b}$ : the dihedral angle of the AI [100] tilt boundary. 
boundary from the melt during fracture stress measurements may have some effect. In order to know this impurity effect, a specimen was wet with the $\mathrm{Sn}-20 \mathrm{Zn}$ melt, followed by the same annealigs as in the dihedral angle measurements and then the fracture stress was measured. The results are shown in $\mathrm{Fig} .4 \mathrm{a}$, indicating the increase in the transition temperature by $\mathrm{Zn}$ and $\mathrm{Sn}$ impurity atoms.

As shown in Fig. 4b, on the other hand, the temperature variation of the dihedral angle also shows the discontinuous changes at nearly the same temperatures, $U$ and $D$, as observed in the fracture stress experiments.

\section{Conclusion}

The relative grain boundary energies of aluminium [100] twist and tilt boundaries have been estimated as a function of the misorientation. The small angle regions of [100] twist and tilt boundary extend to roughly $15^{\circ}$ and $20^{\circ}$, respectively. For the [100] twist boundary, the energy cusps were observed for $\Sigma 61 \mathrm{a}, \sum 65 \mathrm{a}, \Sigma 17 \mathrm{a}, \Sigma 65 \mathrm{~b}, \Sigma 13 \mathrm{a}, \Sigma 5$ and near $\Sigma 29$ a misorientations. For the tilt boundary, the energy cusps were observed at $\sum 61 \mathrm{a}, \sum 65 \mathrm{a}, \sum 13 \mathrm{a}, \sum 65 \mathrm{~b}$ and $\sum 5 \mathrm{misorientations}$ as in the twist boundary. From the misorientation angle with energy cusps, the [100] tilt boundary may be divided into 5 regions.

The fracture stress and the dihedral angle in region $I$ and $\nabla$ abruptly decrease and again abruptly increase with. increasing temperature. These results suggest drastic changes of the boundary energy at these temperatures. Since these change does not observed at $\sum 13$ a misorientation, these transitions show structure changes on the small angle boundaries.

Acknowledgement: We would like to thank Prof. Y. Nakamura for his encouragement and suggestions during the preparation of the manuscript.

References

1. A.P. Sutton and V. Vitek, Phil.Trans.R.Soc., Lond. A309, $1(1983), 1$ $, 37,55$.

2. D. Schwartz, V. Vitek and A.P. Sutton, Phil. Mag. A51 (1985) 499.

3. R.W. Balluffi, Trans. JIM., 27 (1986), Suppl.,23.

4. F. Cosandy and C.L. Bauer, Phil. Mag. A44 (1981), 391.

5. W. Krakow and D.A. Smith, Trans. JIM.,27(1986), suppl.,277.

6. Gui-Jin Wang, A. P. Sutton and V. Vitek, Acta Metal1.,23 (1985), 1113.

7. Gui-Jin Wang and V. Vitek, Acta Metal1., 34(1986),951.

8. A. Otsuki and M. Mizuno, Trans. JIM., 27 (1986), Suppl , 789.

9. N.A. Gjostein and F.N. Rhines, Acta Metall.,7(1959) 319 .

10. Siu-Wai Chan and R.W. Balluffi, Acta Metall.,6(1985),1113.

11. P.D. Bristowe and A.G. Crocker, Phil. Mag., A38(1978), 487 .

12. H. Gleiter, Z. Metallk., 61(1970), 282.

13. W.T. Read and W. Shockley, Phys. Rev., $78(1950), 275$.

14. P.J. Goodhew, Grain Boundary Structure and Kinetics, ed.R.W. Balluffi, ASM, Metals Park, Ohio 1980, p. 155.

15. A.P. Sutton, Phil. Mag., A46 (1982),171.

16. D. Camel, N. Eustathopoulos and D. Desre, Acta Metall., 28(1980), 239. 